\title{
Kernos
}

Revue internationale et pluridisciplinaire de religion grecque antique

$17 \mid 2004$

Varia

\section{Walter BURKERT, Antichità classica e cristianesimo antico. Problemi di una scienza comprensiva delle religioni}

Catherine Lheureux-Godbille

\section{(2) OpenEdition}

Journals

Édition électronique

URL : http://journals.openedition.org/kernos/1514

DOI : 10.4000/kernos. 1514

ISSN : 2034-7871

Éditeur

Centre international d'étude de la religion grecque antique

Édition imprimée

Date de publication : 1 janvier 2004

ISSN : 0776-3824

\section{Référence électronique}

Catherine Lheureux-Godbille, « Walter BURKERT, Antichità classica e cristianesimo antico. Problemi di una scienza comprensiva delle religioni », Kernos [En ligne], 17 | 2004, mis en ligne le 16 juin 2011, consulté le 24 septembre 2020. URL : http://journals.openedition.org/kernos/1514 ; DOI : https:// doi.org/10.4000/kernos. 1514 
Walter BuRKerT, Antichità classica e cristianesimo antico. Problemi di una scienza comprensiva delle religioni, Cosenza, Ed. Lionello Giordano, 2000. 1 vol. 14,5 × 20,5 cm, 193 p. (Biblioteca de Studi Religiosi, 3). ISBN : 88-86919$11-5$

Ce petit ouvrage, qui reprend en traduction italienne le texte de cinq communications scientifiques de Walter Burkert publiées une première fois à Berlin en 1996 sous le titre Klassisches Altertum und antikes Chistentum, a le mérite de remettre dans son contexte l'œuvre de ce chercheur. En effet, la préface de Giovanni Casadio et de Christoph Markschies et, à sa suite, la présentation par ce dernier des lignes conductrices des recherches entreprises par Walter Burkert retracent, à travers une expérience personnelle, les étapes de la constitution de l'histoire des religions en tant que discipline scientifique autonome depuis le début du $\mathrm{xx}^{c}$ siècle, notamment grâce à l'établissement de contacts avec la philologie classique et la théologie. Les travaux de Walter Burkert ont porté principalement sur l'histoire religieuse de l'antiquité, plus spécialement dans la sphère grecque, et ont contribué à délimiter les champs de recherche et à cerner les problématiques d'une science historique tenant compte des religions. La liste des publications de Walter Burkert à la fin de l'ouvrage complète très utilement cet ouvrage de référence.

La première partie de l'ouvrage (p. 21-66) est consacrée à l'examen des relations complexes entre le christianisme et la culture classique depuis la période antique et des conséquences sur le plan scientifique aux $\mathrm{xxx}^{\mathrm{e}}$ et $\mathrm{xx}^{\mathrm{c}}$ siècles. La dimension philologique et culturelle du Nouveau Testament, qui a déterminé dans l'Antiquité tardive une véritable conquête philologico-historique de la part des chrétiens pour établir la visibilité de leur tradition, ne devient un objet d'étude scientifique et d'enseignement qu'aux $\mathrm{xv}^{\mathrm{e}}$ et $\mathrm{xvI} \mathrm{e}^{e}$ siècles avec les humanistes qui fondent leur démarche sur des motivations avant tout religieuses. L'époque des Lumières marque, par ses aspirations à l'autonomie scientifique par rapport à l'Église, l'établissement d'une distance entre l'apprentissage des langues classiques d'une part et la formation générale des clercs d'autre part. À la fin du XVIII" siècle, les travaux d'Edward Gibbon limitent le cadre de recherche sur l'antiquité classique à la période qui s'étend de l'instauration du régime démocratique athénien au $\mathrm{v}^{\circ}$ siècle au règne d'Auguste, amorçant ainsi très clairement un processus de séparation de la philologie et de la théologie qui se prolonge durant tout le $\mathrm{xxx}^{\prime \prime}$ siècle. C'est à cette époque que se développent les premières initiatives en matière d'histoire des religions. Parmi elles, il faut noter les éditions de textes d'auteurs ecclésiastiques grecs et latins et les contributions des différentes écoles scientifiques européennes. Dans la première moitié du $\mathrm{xx}^{*}$ siècle, les travaux de Hans Lietzmann (1875-1942), théologien de formation, professeur à Gènes de 1905 à 1924 et successeur d'Aclolf von Harnack à l'Académie de Berlin à partir de 1927, jouent un tôle de premier plan dans le développement de l'histoire des religions en tant que discipline scientifique à part entière. En conclusion, il est intéressant de constater à quel point l'augmentation du nombre des documents mis à clisposition des chercheurs a posé davantage de problèmes qu'elle n'en a réglés pour l'établissement d'une histoire des religions. Toutefois, malgré leurs dérives, les différentes démarches ont abouti aujourd'hui à la délimitation d'un domaine scientifique où la victoire du christianisme n'est plus considérée comme un progrès évident et où l'humain est replacé clans un cadre résolument multiculturel.

La deuxième contribution (p. 67-92) aborde le problème de la religiosité personnelle de Plutarque. À la lecture, son ouvvre vaste donne l'impression de connaitre intimement l'auteur qui y développe des spéculations religieuses et philosophiques. Il 
s'agit non seulement d'un exposé théologique sous la forme de la littérature traditionnelle, mais aussi de l'expression, allusive mais bien réelle, d'espérances personnelles. Prêtre à Delphes et officiant pour les cultes de la cité de Chéronée, Plutarque exprime ses convictions par rapport à l'importance de la fonction de la communauté dans la pratique religieuse.

La troisième communication (p. 93-116) est consacrée à un état des recherches à propos de l'importance respective des influences phéniciennes et anatoliennes sur la culture grecque. L'A. pose un regard critique sur les différentes interventions de Santo Mazzarino : replaçant les éléments de la problématique dans un large contexte idéologique, il conclut à l'importance des courants d'Asie mineure, notamment indoeuropéens, sur les populations grecques depuis l'époque archaïque mais surtout au rôle prépondérant de l'activité maritime des Grecs depuis le viII" siècle dans le processus de stimulation culturelle.

La quatrième partie (p. 117-150) appréhende la conception de l'être humain intérieur dans les doctrines platonicienne et paulinienne. Encore aujourd'hui, les progrès de la médecine n'ont pas fait disparaître la croyance en l'existence d'une âme à l'intérieur du corps qui constituerait une personne interne distincte de la personne corporelle. La présence de formules de ce genre dans la philosophie platonicienne et dans la tradition chrétienne démontre non seulement la persistence du concept de corps et d'âme, mais également son adaptation aux contextes historico-culturels. Immortelle et manifestée de manière psycho-somatique chez Homère, l'âme chez Platon apparaît non seulement séparable du corps, mais également immatérielle et incorporelle au sens le plus profond, c'est-à-dire inaccessible aux sens et impossible à localiser dans aucune partie, intérieure ou extérieure, du corps. Le concept a été repris et développé par d'autres auteurs : Sénèque, Marc Aurèle, Porphyre. Cette communication est la seule des cinq contributions reprises dans ce volume à ne pas comporter de bibliographique finale récapitulative, mais les notes de bas de page compensent avantageusement cette absence.

La cinquième et dermière partie (p. 151-178) aborde le problème de la violence dans l'histoire religieuse à travers la répression du manichéisme. Malgré la valeur incontestable accordée au langage, force est de reconnaître l'existence d'un espace de violence à l'intérieur des sociétés humaines antiques: la violence est légitimée, notamment dans sa forme pénale. Fondé sur le scandale créé par l'exécution d'un innocent, le message chrétien est articulé paradoxalement sur le rejet de toutes les formes de violence et d'agression, et sur l'annonce d'une paix chantée par une armée céleste et gagnée au prix d'un combat final contre les démons. L'infléchissement de la politique impériale sous le règne de Constantin va normaliser le recours à la violence pénale en faveur du christianisme, notamment dans le cadre de la lutte contre les dissidents religieux, comme les manichéens.

Catherine Lheureux-Godbille (FNRS - Université de Liège)

Angelo BreliCH, Mitologia, politeismo, magia e altri studi di storia delle religioni (1946-1977), a cura di Paolo Xella, Napoli, Liguori Editore, 2002. 1 vol. $16 \times 24 \mathrm{~cm}$, XII +182 p. (Anthropos, 38). ISBN : 88-207-3415-X.

Paolo Xella publie un recueil des travaux de l'historien des religions italien Angelo Brelich (1913-1977), vingt-cinq ans après la mort de celui-ci. Comme il le précise dans la préface, ce livre réunit les articles de Brelich parus dans la revue Studi e materiali di storia delle religioni - dont Brelich était l'éditeur -, ainsi que ceux qui furent présentés 